\title{
Postsurgical prescriptions for opioid naive patients and association with overdose and misuse: retrospective cohort study
}

\author{
Gabriel A Brat, ${ }^{1,2}$ Denis Agniel, ${ }^{1}$ Andrew Beam, ${ }^{1}$ Brian Yorkgitis, ${ }^{3}$ Mark Bicket, ${ }^{4}$ Mark Homer, ${ }^{1}$ \\ Kathe P Fox, ${ }^{5}$ Daniel B Knecht, ${ }^{5}$ Cheryl N McMahill-Walraven, ${ }^{5}$ Nathan Palmer, ${ }^{1}$ Isaac Kohane ${ }^{1}$
}

${ }^{1}$ Department of Biomedical Informatics, Harvard Medical School, Countway Library, Boston, MA 02215, USA

${ }^{2}$ Department of Surgery, Beth Israel Deaconess Medical Center, Boston, MA, USA ${ }^{3}$ Department of Surgery, University of Florida, Jacksonville, Division of Acute Care Surgery, University of Florida College of MedicineJacksonville, Jacksonville,

FL, USA

${ }^{4}$ Department of Anesthesia and Critical Care Medicine, Johns Hopkins University, Baltimore, MD, USA

${ }^{5}$ Department of Analytics and Behavior Change, Aetna, Blue Bell, PA, USA

Correspondence to: G A Brat gbrat@bidmc.harvard.edu

Additional material is published online only. To view please visit the journal online.

Cite this as: $B M J$ 2018;360:j5790 http://dx.doi.org/10.1136/bmj.j.5790

Accepted: 1 December 2017

\section{ABSTRACT}

OBJECTIVE

To quantify the effects of varying opioid prescribing patterns after surgery on dependence, overdose, or abuse in an opioid naive population.

DESIGN

Retrospective cohort study.

SETTING

Surgical claims from a linked medical and pharmacy administrative database of 37651619 commercially insured patients between 2008 and 2016.

\section{PARTICIPANTS}

1015116 opioid naive patients undergoing surgery.

\section{MAIN OUTCOME MEASURES}

Use of oral opioids after discharge as defined by refills and total dosage and duration of use. The primary outcome was a composite of misuse identified by a diagnostic code for opioid dependence, abuse, or overdose.

RESULTS

568612 (56.0\%) patients received postoperative opioids, and a code for abuse was identified for 5906 patients $(0.6 \%, 183$ per 100000 person years). Total duration of opioid use was the strongest predictor of misuse, with each refill and additional week of opioid use associated with an adjusted increase in the rate of misuse of $44.0 \%$ (95\% confidence interval $40.8 \%$ to $47.2 \%, \mathrm{P}<0.001)$, and $19.9 \%$ increase in hazard ( $18.5 \%$ to $21.4 \%, \mathrm{P}<0.001)$, respectively.

\section{CONCLUSIONS}

Each refill and week of opioid prescription is associated with a large increase in opioid misuse among opioid naive patients. The data from this study suggest that duration of the prescription rather than dosage is more strongly associated with ultimate misuse in the early postsurgical period. The analysis quantifies the association of prescribing

\section{WHAT IS ALREADY KNOWN ON THIS TOPIC}

\section{Opioid misuse is increasing rapidly in the US and internationally}

Surgical patients are four times more likely to get opioids at discharge than their non-surgical counterparts

It is unknown how opioid prescribing habits by clinicians are related to rates of misuse

\section{WHAT THIS STUDY ADDS}

Each refill and additional week of opioid prescription is associated with a large increase in opioid misuse among opioid naive patients

The duration of a prescription rather than opioid dosage was more strongly associated with ultimate misuse in the early postsurgical period choices on opioid misuse and identifies levers for possible impact.

\section{Introduction}

Since the early 2000s, age adjusted rates of opioid overdose have tripled and now rank as the leading cause of death related to unintentional injury. ${ }^{12}$ Prescription drugs are implicated in most of the cases, as rates of opioid prescription quadrupled ${ }^{34}$ and were paralleled by increasing rates of deaths from overdose. Non-fatal overdose events from prescription opioids account for 7-11 times more episodes than fatal overdoses ${ }^{25}$ and have similarly increased by more than $50 \%$ over 10 years. $^{6}$ Strikingly the majority of these non-fatal overdose episodes take place in patients identified as non-chronic (<90 days) opioid users.

Overprescribing of opioids is thought to be a major contributor, ${ }^{7}$ where two thirds of opioid misuse can be attributed to opioids obtained through a single physician. ${ }^{2}$ Overprescribing enables opioid diversion and increases the potential for addiction. ${ }^{8}$ 9 Surgical patients are nearly four times more likely to get post-discharge opioids than their nonsurgical counterparts. Orthopedic surgeons alone were responsible for $7.7 \%$ of opioid prescriptions in 2009. ${ }^{1011}$ Despite these numbers, surgeons have yet to find the right balance of opioid prescriptions: between $3 \%$ and $10 \%$ of opioid naive patients become chronic users, and emerging research suggests that as many as $80 \%$ of prescribed pills in the remaining group of patients are unused. ${ }^{12}$

The lack of guidance around postsurgical opioid prescribing $^{13} 14$ partially results from little being known about the effect of longer and larger regimens of post-discharge opioids. More directly, it is not known how prescription refills affect the long term likelihood of misusing opioids. Prominent authors have called for study into this issue ${ }^{61516}$ to underpin future guidelines. ${ }^{17}$ Furthermore, evidence shows that any post-discharge use is a risk factor for multiple refills ${ }^{18}$ independent of the specific prescription. ${ }^{19}$ In this study, we examined the association between opioid prescription refills after surgery and misuse in an opioid naive population.

\section{Methods}

\section{Data source}

We extracted information on surgical patients with medical and pharmacy insurance from a deidentified administrative database at Aetna, a commercial managed healthcare company. This database includes 37651619 members with Aetna health and pharmacy insurance coverage between 2008 and early 2016. 
Data included all medical and pharmacy claims during the study period.

\section{Sample cohort}

For this retrospective cohort study, the sample consisted of members who underwent surgery and had at least six months of medical insurance and three months of pharmacy insurance before surgery, as well as 90 days of pharmacy coverage and one year of medical coverage ${ }^{16}$ after surgery. For the index surgery we chose the first surgery for each database member that met criteria and after which no further surgery claims were filed for 90 days. Members were followed until they experienced an opioid related event or their last month of enrollment in the database.

A member was considered opioid naive and eligible for inclusion if his or her total opioid use in the 60 days before surgery was seven days or less. ${ }^{20}$ Postsurgical opioid use was measured if the member filled a prescription for an included opioid within 30 days of discharge. Use was considered to have stopped when either 30 days elapsed without a filled opioid prescription or a misuse diagnosis was observed.

We excluded patients with presurgical evidence of opioid or other non-specific forms of misuse in the six months before surgery (see supplemental eTable 1 for a list of presurgical exclusion codes). Finally, we excluded a small subset of patients with missing data for any variable. Supplemental eFigure 1 summarizes the protocol and derivation of the sample.

\section{Outcome measures}

Surgical claims were identified by a comprehensive list of Current Procedural Terminology (CPT) codes associated with inpatient and outpatient surgery and specialty released by the National Surgical Quality Improvement Program of the American College of Surgeons in 2015. ${ }^{21}$ Organ based categories were derived from top level CPT headers (eg, 10030-19499 for surgeries of the integumentary system).

The primary outcome was an ICD-9 (international classification of diseases, ninth revision) diagnosis code of opioid dependence, abuse, or overdose (see supplemental eTable 1). Opioid misuse was defined as the presence of at least one of these ICD codes after discharge and encompasses a composite of a wide range of forms of misuse. We included only diagnosis codes related specifically to prescription opioids.

\section{Opioid use}

Opioids were identified in the database as narcotic analgesics or narcotic analgesic combinations by therapeutic category from Cerner's Multum Lexicon Drug Database. ${ }^{22}$ We used only non-injected drugs associated with the following primary ingredients: codeine, hydrocodone, hydromorphone, morphine, oxycodone, oxymorphone, and tramadol. Other less common opioids were excluded for low numbers or association with palliative care or dependence treatment. We determined the morphine milligram equivalent (MME) dosage for each opioid prescription, using standard conversions. ${ }^{23}$ To decrease the influence of extreme outliers while respecting variation in the literature, we truncated the daily dose at $350 \mathrm{MME} /$ day. ${ }^{24}$ Length of use was truncated at 90 plus one days, and number of refills was truncated at five.

\section{Refill identification}

A drug refill is a physical event of varying durations. As with the initial prescription, there is no standard refill dosage or duration. Thus, identification of the event is an artificial threshold marking continuation of opioid use. The event is also relevant because it requires patients to approach their care provider for further drugs. We therefore chose to identify refills in two ways. First, we counted the number of physical prescriptions filled after initial use. The first postdischarge prescription was counted as the initial use and we included all subsequent prescriptions with less than a 30 day gap between prescriptions. Second, we identified total post-discharge use by duration and dosage. We determined the post-discharge duration of opioid use to be the total number of calendar days covered by a prescription for an opioid after discharge from the index surgical procedure. This identified the "cabinet supply" of opioids acquired by patients. ${ }^{25} \mathrm{We}$ used a well described method ${ }^{26}$ to consider overlapping prescriptions as part of the same episode and an indication of a completed previous prescription at a higher dose. Accounting for overlapping prescriptions consisted of defining use as the total number of days of accumulated prescriptions minus overlap.

When a single discharge date was listed on all medical claims associated with the index surgery date, we used this as the official discharge date. When multiple discharge dates were present, we used the last date. If no discharge date was associated with any medical claim on the index surgery date, we used the surgery date as discharge date.

\section{Statistical analysis}

We analyzed the time until a misuse event over the entire study period. To compute raw rates of opioid misuse we used the total number of misuse events divided by total follow-up time. These are reported as cases per 100000 person years. Weighted linear regression was used for unadjusted analysis of log transformed weekly rates of misuse, where each week was weighted according to sample size. For adjusted analysis of time until misuse event we used Cox proportional hazards models. Adjusted models included either refills or duration, as well as daily dose (MME/day), age, sex, US state of residence, surgery type by CPT top level grouping, surgery year, concurrent benzodiazepine use, and binary indicators of presurgical diagnoses potentially related to misuse. A single surgery might be associated with multiple surgery types, if multiple CPT codes were assigned.

We used penalized logistic regression to determine presurgical diagnoses of interest.27 In the model we included all 590 ICD-9 codes assigned to at least 0.5\% of patients in the six months before surgery, as well 
$e^{\eta_{i}} /\left(1+e^{\eta_{i}}\right)$ where $\eta_{i}=-2.5+d_{i}+\log (5) \times m$

$d_{i}$ is the duration of exposure for patient $i$ and $m_{i}$ is an indicator of misuse

Fig 1 | Mean of Bernoulli random variable generating synthetic binary confounder

as age, sex, and surgery type. In total, we selected 65 presurgical diagnosis codes (see supplemental eTable 2).

Weighted linear regression effects are reported as multiplicative percentage increases in rate, and Cox effects as multiplicative percentage increases in hazard or equivalently hazard ratios. Throughout we report two sided $\mathrm{P}$ values and 95\% confidence intervals. All analyses were conducted using R 3.2.2 (R Core Team).

To ensure that the effect we observed was driven by initial opioid use and not downstream unidentified factors, we performed sensitivity analyses restricted to one year postsurgery and to members with no additional surgeries during follow-up. For misuse events within one year, we used logistic regression to adjust for covariates.

To detect if structural factors owing to changing trends over time (year of surgery) or geography were influencing our estimates, we considered two additional sensitivity analyses: a Cox model including an interaction between duration and an indicator for year and a model including an interaction between duration and state of residence. Further sensitivity analysis attempted to mimic an unobserved confounder by creating a synthetic binary variable that was associated with both duration and opioid misuse. The binary variable was generated as a Bernoulli random variable (fig 1). To determine the extent to which strong unobserved confounding might explain the observed association, we included this synthetic confounder in a Cox model.

\section{Patient involvement}

No patients were involved in setting the research question or the outcome measures, nor were they involved in developing plans for design or implementation of the study. No patients were asked to advise on interpretation or writing up of results. There are no plans to disseminate the results of the research to study participants or the relevant patient community.

\section{Results}

\section{Cohort characteristics}

The study sample included 1015116 members who met study criteria and underwent an index surgery. Members were followed for a median of 2.67 years. After the index surgery, 568612 members (56.0\%) filled a prescription for a postoperative opioid. Ninety per cent of prescriptions were filled within three days of discharge. In the subsequent follow-up period, misuse was identified in 5906 members $(0.6 \%, 183$ cases per 100000 person years), with 1857 occurring within one year after surgery (0.2\%).

Characteristics of the cohort followed national trends (table 1). Surgeries were more prevalent among older age groups, and younger groups had higher rates of opioid usage. The most common surgeries were for the musculoskeletal system (367317 surgeries; 2448 misuse events; 206 cases per 100000 person years), digestive system (293905 surgeries; 1825 misuse events; 198 cases per 100000 person years), and integumentary system (106914 surgeries; 533 misuse events; 161 cases per 100000 person years). Rates of misuse by age group followed national patterns, with higher rates among younger men (fig 2A) and increasing rates over the study period (fig 2B).

Over the study period there were notable changes in the characteristics of opioid prescriptions and the rates of misuse (table 1). Postsurgical incidence of misuse increased from 183 cases per 100000 person years (2009) to 269 cases per 100000 person years (2014), whereas rates for opioid prescription fill plateaued and began to fall in the later years of the study (also see fig 2C). Median duration and median dose prescribed remained stable throughout the study period at about five days and $50 \mathrm{MME} /$ day, respectively. These stable numbers masked a change in opioid prescription characteristics during the study period: fewer short course and increased numbers of longer duration prescriptions as well as a trend toward lower doses by episode (see figs 3B and 3D). Similar prescribing changes were detected for all surgery types (see supplemental eFigure 2).

\section{Rates of misuse by opioid use}

The number of post-discharge prescriptions best predicted eventual misuse. Overall rates of misuse were low, but rates grew rapidly with increasing opioid use. The rate of misuse more than doubled among those with one refill (86 654 (15.2\%); 293 cases per 100000 person years) versus those with no refills (434273 (76.2\%); 145 cases per 100000 person years). In total, each additional refill increased the rate of misuse by $70.7 \%$ (95\% confidence interval $54.6 \%$ to $88.4 \%$ ) before adjustment and increased the hazard of misuse by $44.0 \%(40.8 \%$ to $47.2 \%, \mathrm{P}<0.001)$ after adjusting for covariates.

The relation between number of refills and misuse was further supported by evaluation of the number of days of opioid use post-discharge. Each additional week of opioid use was associated with an average increase in the rate of misuse of $34.2 \%$ (26.4\% to $42.6 \%, \mathrm{P}<0.001$, see fig $3 \mathrm{~A})$. Adjusting for covariates, each additional week of opioid use was associated with a 19.9\% increase in hazard (95\% confidence interval $18.5 \%$ to $21.4 \%, \mathrm{P}<0.001$ ). For both refills and duration, the risk of misuse initially follows the trend line and begins to taper at higher levels of use greater than 11 weeks (fig 3A).

Compared with duration of use, the dosage prescribed was a weaker predictor of misuse (fig 3C), 


\begin{tabular}{|c|c|c|c|c|c|c|c|c|}
\hline Characteristics & Total & $\begin{array}{l}\text { Misuse/ } \\
\text { overdose } \\
\text { events }\end{array}$ & $\begin{array}{l}\text { Opioid } \\
\text { prescription } \\
\text { filled (\%) }\end{array}$ & $\begin{array}{l}\text { Median } \\
\text { duration } \\
\text { (days) }\end{array}$ & $\begin{array}{l}\text { Median } \\
\text { MME/day }\end{array}$ & $\begin{array}{l}\text { Median } \\
\text { follow-up } \\
\text { (years) }\end{array}$ & $\begin{array}{l}\text { Median } \\
\text { time to } \\
\text { misuse } \\
\text { (years) }\end{array}$ & $\begin{array}{l}\text { Cases per } \\
100000 \\
\text { person } \\
\text { years }\end{array}$ \\
\hline Total sample & 1015116 & 5906 & 56 & 5 & 50 & 2.67 & 1.74 & 183.2 \\
\hline Female & 563170 & 3166 & 54.3 & 5 & 50 & 2.67 & 1.82 & 176.3 \\
\hline Male & 451946 & 2740 & 58.2 & 5 & 50 & 2.66 & 1.67 & 191.8 \\
\hline \multicolumn{9}{|l|}{ Age group (years): } \\
\hline$<15$ & 54098 & 118 & 46 & 5 & 30 & 2.74 & 2.39 & 66.7 \\
\hline $15-24$ & 92458 & 1160 & 62.9 & 5 & 54.2 & 2.51 & 1.47 & 420.6 \\
\hline $25-34$ & 97856 & 666 & 60.6 & 5 & 50 & 2.41 & 1.68 & 226 \\
\hline $35-44$ & 175969 & 1086 & 58.9 & 5 & 50 & 2.69 & 1.9 & 190.7 \\
\hline $45-54$ & 238154 & 1284 & 57.9 & 5 & 50 & 2.78 & 1.95 & 164 \\
\hline $55-64$ & 211308 & 989 & 56.1 & 5 & 50 & 2.55 & 1.64 & 152.4 \\
\hline$\geq 65$ & 145273 & 603 & 45.5 & 5 & 45 & 2.93 & 2.07 & 126.9 \\
\hline \multicolumn{9}{|l|}{ Surgery year: } \\
\hline 2008 & 142332 & 1031 & 47.5 & 5 & 50 & 3.3 & 2.76 & 183.1 \\
\hline 2009 & 205618 & 1374 & 53 & 5 & 50 & 3.62 & 2.51 & 169.5 \\
\hline 2010 & 157640 & 982 & 61.1 & 5 & 50 & 3.66 & 2.28 & 168.9 \\
\hline 2011 & 137648 & 780 & 62.8 & 5 & 50 & 3.52 & 1.72 & 171.6 \\
\hline 2012 & 130096 & 705 & 57.6 & 5 & 50 & 3.2 & 1.38 & 193 \\
\hline 2013 & 113841 & 505 & 63.9 & 5 & 50 & 2.39 & 1.29 & 195 \\
\hline 2014 & 110392 & 461 & 49.1 & 5 & 50 & 1.52 & 0.76 & 268.7 \\
\hline \multicolumn{9}{|l|}{ Surgery type: } \\
\hline Musculoskeletal & 367317 & 2448 & 60.3 & 6 & 57.7 & 2.71 & 1.86 & 206.2 \\
\hline Gastrointestinal & 293905 & 1825 & 63.2 & 5 & 50 & 2.63 & 1.68 & 198.1 \\
\hline Integumentary & 106914 & 533 & 39.3 & 5 & 45 & 2.61 & 1.75 & 160.8 \\
\hline Gynecological & 98444 & 449 & 56.7 & 5 & 46.9 & 2.69 & 1.85 & 142.3 \\
\hline Cardiovascular & 57715 & 241 & 33.3 & 6 & 44.4 & 2.65 & 1.52 & 134.4 \\
\hline Neurological & 38698 & 374 & 61.1 & 8 & 56.2 & 2.6 & 1.52 & 306.5 \\
\hline Renal & 30274 & 121 & 49.8 & 5 & 45 & 2.71 & 1.52 & 125.8 \\
\hline Male genital & 26524 & 76 & 59.3 & 5 & 41.2 & 2.73 & 1.98 & 89.6 \\
\hline Endocrine & 19622 & 110 & 49.2 & 4 & 45 & 2.72 & 1.6 & 172 \\
\hline Hernia and lymphatic systems & 11836 & 56 & 62.6 & 5 & 50 & 2.6 & 1.52 & 153.9 \\
\hline Auditory & 10250 & 37 & 30.1 & 5 & 41.7 & 2.62 & 1.76 & 114.6 \\
\hline Respiratory & 6280 & 50 & 59.5 & 8 & 50 & 2.44 & 0.85 & 270.2 \\
\hline Maternity care and delivery & 2692 & 13 & 67.4 & 4 & 46.9 & 2.42 & 2.28 & 162.2 \\
\hline Mediastinum and diaphragm & 1186 & 13 & 62 & 6 & 67.5 & 2.74 & 2.52 & 344.1 \\
\hline Reproductive & 10 & 0 & 30 & 5 & 30 & 1.51 & - & 0 \\
\hline \multicolumn{9}{|l|}{ Drugs: } \\
\hline Hydrocodone & 275292 & 1695 & - & 5 & 45 & 2.83 & 1.95 & 187.5 \\
\hline Oxycodone & 205559 & 1432 & - & 5 & 62.5 & 2.7 & 1.68 & 219 \\
\hline Codeine & 35041 & 148 & - & 4 & 27 & 2.84 & 2.45 & 127.6 \\
\hline Mixed & 30803 & 324 & - & 9 & 60 & 2.62 & 1.52 & 339.7 \\
\hline Tramadol & 11721 & 70 & - & 8 & 30 & 2.21 & 1.53 & 221.1 \\
\hline Hydromorphone & 9600 & 123 & - & 5 & 66.7 & 2.47 & 1.45 & 427.6 \\
\hline
\end{tabular}

and dose became important only with extended use (fig 3E). Each additional $10 \mathrm{MME} /$ day was associated with only a $0.8 \%$ increase in hazard of misuse (adjusted hazard ratio 1.008, 95\% confidence interval 1.003 to $1.013, \mathrm{P}=0.001)$. Even high doses ( $>150$ MME/day) were associated with only mild increases in risk when duration was short (fig 3E). For example, when the duration of post-discharge prescription was less than two weeks, similar rates of misuse were found for lower (40-50 MME/day) compared with higher (100-150 MME/day) opioid dose. Conversely, members receiving greater than nine weeks of opioids at a higher dose had dramatically increasing rates of misuse: 476 cases per 100000 person years at $<20$ MME/day $(n=422)$ to 2398 cases per 100000 person years at 50-60 MME/day ( $\mathrm{n}=430)$ to 5689 cases per 100000 person years at $>150 \mathrm{MME} /$ day $(\mathrm{n}=237)$. For short term opioid use less than 90 days, higher doses of opioids had smaller effects on the rate of misuse than did additional weeks of use.

\section{Additional risk factors of misuse}

After adjusting for covariates, other risk factors (see supplemental eTable 2), including benzodiazepines (hazard ratio 1.77, 95\% confidence interval 1.64 to 1.93) as well as regimens initiated with hydromorphone (1.76, 1.37 to 2.26$)$ and oxycodone $(1.24,1.03$ to 1.48$)$ had a statistically significant association with opioid misuse. The adjusted effect of surgery was greatly attenuated after controlling for the strongly associated presurgical diagnoses. Notable presurgical diagnoses included bariatric surgery status (V45.86; hazard ratio $2.19,95 \%$ confidence interval 1.77 to 2.72 ), tobacco use disorder $(305.1 ; 2.16,1.97$ to 2.36$)$, other chronic pain (338.29; 2.02, 1.68 to 2.42$)$, and major depressive disorder $(311 ; 1.60,1.44$ to 1.78$)$. 

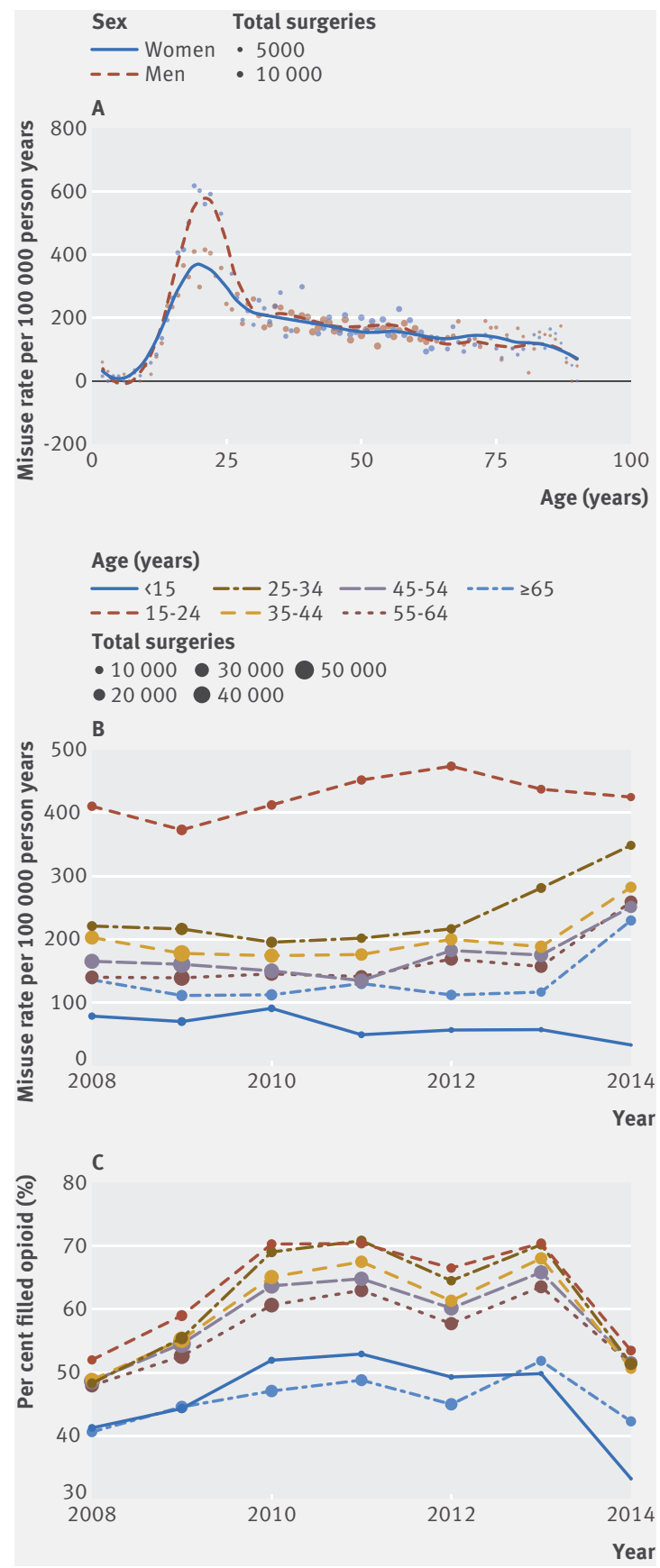

Fig 2 | Misuse of and prescription rates for opioids per 100000 person years across ages and sex over time by cohort

\section{Sensitivity analyses}

As part of a sensitivity analysis, we constructed models that removed potential confounders. We found no difference in effect, with similar results for misuse events within one year of surgery (see supplemental eFigure 3) and among patients with no additional surgery during follow-up (see supplemental eFigure 4). To ensure that our outcome analysis was not biased by a specific ICD-9 code, we removed opioid dependence code 304.00 , the most common code, leaving only specific abuse and overdose codes. The findings of the model were virtually unchanged with this smaller subset: each additional refill was associated with an increased risk of $70.9 \%$ versus $70.7 \%$ in the comprehensive model.

We also verified that the observed association was not affected by geography or biased by changing conditions across the study period. We compared the association between duration and misuse over different years (fig 4C) and at the state level; results were statistically indistinguishable from a model with aggregated duration effect (likelihood ratio test $\mathrm{P}=0.26$ and $\mathrm{P}=0.99$, respectively). Figure $4 \mathrm{~A}$ shows that surgeons appear to have reduced the mean dosage within their specialty during the study period. Typical reductions ranged from 3 to $18 \mathrm{MME} /$ day (4\% to 24\%) over the duration of the study. While opioid dosage fell, mean duration of use during the years of the study remained relatively stable (fig $4 \mathrm{~B}$ ). Despite changing clinician behavior over time, the relation between duration of use and misuse was persistent (fig 4C, note that only weeks with $>100$ surgeries are included). Such stability is further evidence of the robustness of this effect.

Finally, we assessed the potential effect of unobserved confounding by generating a synthetic binary variable strongly associated with both duration of use (odds ratio 2.7) and misuse (odds ratio 5.0, see fig 1) and inserted it into the model. An example of such a confounder could be an undiagnosed risk factor for postsurgical misuse, such as presurgical alcohol dependence. Even in the presence of this artificial explanatory confounder, which has an unrealistically strong relation to misuse, each week of use was still associated with a 13\% increase in hazard of misuse.

\section{Discussion}

Physicians struggle to appropriately prescribe and dose postoperative opioids while tackling the real needs of acute pain from surgery. ${ }^{28} 29$ This study quantifies the strong relation between number and duration of refills of prescribed opioid drugs and subsequent opioid misuse in the surgical population. We focused on typical surgical patients without a history of misuse or ongoing opioid use and estimated an adjusted $44 \%$ increase in misuse for every refill fulfilled, or $20 \%$ increase for every week of prescription. This association remained statistically significant in multiple sensitivity analyses and using both time until any event and events within one year of surgery. Although rates of misuse were low, the large number of surgeries performed every year increases the importance of these numbers.

Our adjusted models suggest that the effect of duration is not explained by temporal changes in physician behavior or by patient population. By 2014, the number of patients receiving opioids and the number of patients with prescriptions of short durations ( $<4$ days) appears to have been reduced. Clinicians increased rates of prescriptions of longer durations for a subset of patients (see fig 3B). In the face of these changes, overall rates of opioid misuse 

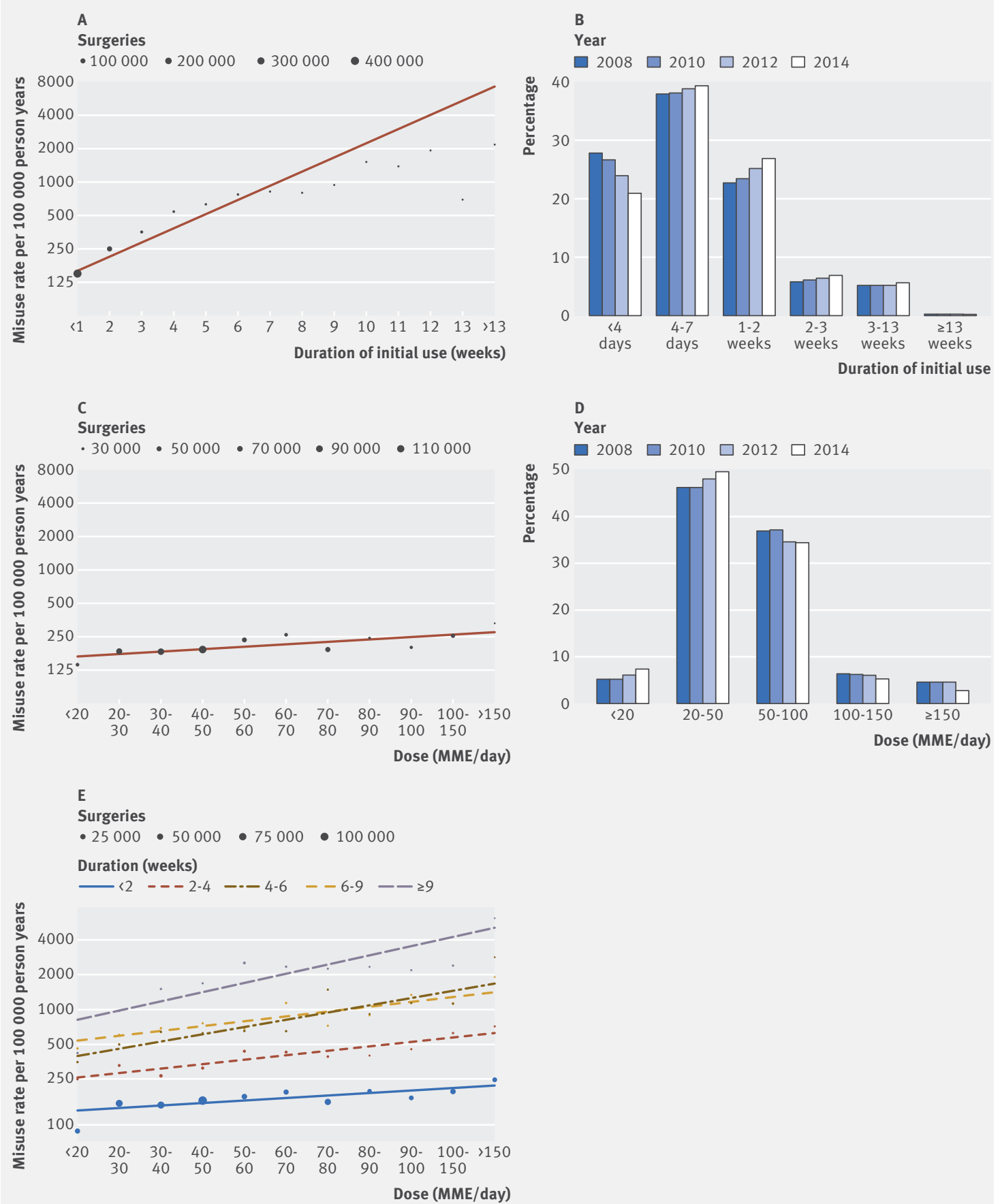

Fig 3 | Postsurgical rates of misuse by dosage and duration of opioid prescription. $M M E=$ morphine milligram equivalent

have continued to increase (fig 2B), showing that this crisis is multifactorial and not only driven by duration of use at an aggregate level. But despite a worsening trend and temporal changes, we found that the effect of duration was stable across study years and was unchanged by evolving misuse and prescribing rates in the population. The stable relation is suggestive of an independent effect (fig 4C).

A second finding was that duration of use rather than dosage of equivalent opioids was more strongly associated with subsequent misuse of post-discharge prescriptions. This builds on previous findings 3031 showing the importance of prescription duration. Each week of opioid use was associated with a $20 \%$ increase in misuse; short term dosage carried a small (about 10\%) incremental impact per 100 morphine milligram equivalent (MME) on misuse and became noteworthy only at longer durations of prescription. While this seems discordant with other studies that found 2-9-fold increases in rates of misuse for doses 

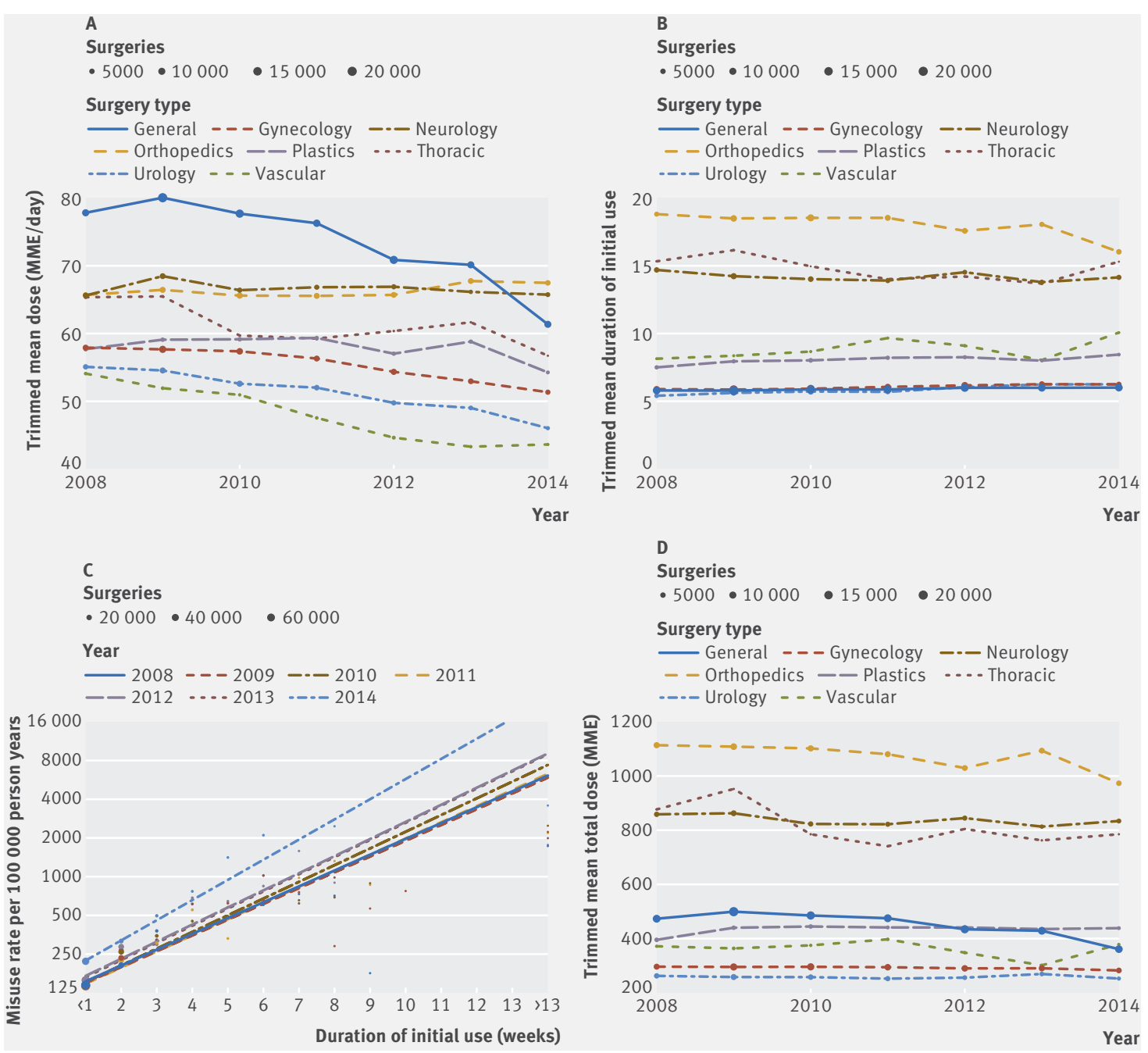

Fig 4 | Temporal effects and sensitivity analysis for opioid use. MME=morphine milligram equivalent

greater than $100 \mathrm{MME} / \mathrm{day},{ }^{572431}$ our work differs from these studies-which focused on chronic users-by examining a population of general surgical patients who typically receive opioids for fewer than two weeks. Patients with chronic opioid usage may exhibit different risk profiles for misuse.

\section{Comparison with other studies}

Our data were consistent with the literature: the rate of refills ${ }^{32}$ and the misuse event rate of $0.2 \%$ within one year $^{33}$ was similar to those identified in other studies. These numbers build on recent literature about the broad effect of post-discharge prescriptions on subsequent opioid use. Patients who received even one post-discharge prescription were three times more likely to be taking opioids at one year. ${ }^{18}$ This finding extended across specialties, where surgical and nonsurgical patients had similar rates of opioid refills. Several studies in surgical patients have also shown that administering opioids early after surgery is associated with subsequent long term usage. ${ }^{31} 34$ Irrespective of the direction of causality, our data suggest that patients who require subsequent refills of opioid drugs are statistically more likely to have an episode of misuse, even years after the index surgery. Whether driven by the patient's underlying need or the clinician's tendency to prescribe opioids, this relation further holds when examining refills as individual weeks of use.

Our findings suggest that opioid naive patients who receive low to moderately high doses of analgesics for short durations have small associated increases in overall rates of misuse. Many studies have shown that pain is often poorly managed after surgery. ${ }^{35-39}$ Higher doses within standards of moderation may better saturate $\mu$ receptors, whereas under-treatment of acute pain increases the risk of pseudoaddiction, chronic pain, and, potentially, overdose. ${ }^{40} 41$ These findings suggest a more nuanced understanding of the relation between duration and dosage, with a focus on early appropriate treatment of pain (including higher doses) for a limited time. Such findings imply that optimal postoperative prescribing, which maximizes analgesia and minimizes the risk of misuse, may be achieved with moderate to high opioid dosages at shorter durations, a combination that merits further investigation in population based and clinical studies. ${ }^{41}$ 


\section{Limitations of this study}

We recognize that administrative data have inherent biases that may have affected our results. First, the dataset does not exclude patients with undocumented presurgical misuse or opioid usage. Similarly, postoperative misuse might not have been detected in members who left the cohort because of lost or changed coverage. Miscoded claims are possible but less likely as coding of opioid abuse has been found to be accurate $85 \%$ of the time. ${ }^{42}$ Alternatively, increased recognition of the problem of opioid misuse may lead to overcoding in later years or undercoding in earlier years. Overcoding could explain the increasing rates of misuse observed in later years, but recent national studies by other authors have also shown similar trends. ${ }^{1}$ Finally, measurement of opioid use is complicated by the possibility that patients might fill a prescription and modify the course or dosing of the drug. ${ }^{43}$ We used the cabinet supply method for measuring use in an attempt to conservatively overestimate usage.

As for the problem of confounding, although we controlled for disease burden by adjusting for surgery type and examined the full spectrum of presurgical diagnosis codes, these are, at best, partial measures of disease state at the time of surgery. Notably, we were unable to control for the extent of pain or the individual features of surgical techniques. In the presence of uncontrolled confounding, we cannot be certain of the magnitude of the effect we observed. Those patients with a higher likelihood of developing misuse might request augmented treatment. ${ }^{44}$ The consistency of our findings, despite extensive sensitivity analyses, suggests there may yet be a causal component to our analysis. This is further supported by evidence linking most patients who present to addiction centers to an initial prescribed opioid for pain. ${ }^{45}$

Finally, the generalizability of this study is limited to insured adults in the US, as several studies have shown increased rates of misuse in Medicaid, Medicare, and veteran populations. ${ }^{254647}$

\section{Conclusions and policy implications}

In this study, we quantified the strong association between short term postsurgical refills and misuse. A single refill increased the potential of misuse by more than $40 \%$, and the duration of use appeared to be the most prominent predictor of misuse. Our findings are important as they offer a potential lever for intervention and behavior change after surgery. Given that surgical and non-surgical patients receive similar numbers of opioid refills, these findings have the potential to extend beyond surgery. Surgeons and non-surgeons are changing the characteristics of their opioid prescriptions, but rates of misuse continue to increase. Clinicians are trapped between guidelines that recommend shorter duration and lower dosing of opioid drugs and a subset of patients who request or require opioids beyond the initial prescription. With these seemingly conflicting forces at play, our analysis provides a broad evidentiary framework to inform clinician behavior and promote protocol development. Further research of this relation is needed to determine how initial treatment regimens can minimize misuse and addiction.

We thank Aetna for donating the dataset. MB was supported by the National Institute of General Medical Sciences of the National Institutes of Health (award No T32GM075774).

Contributors: GAB, DA, and MB designed the study. CNMCM-W, DBK, and KPF contributed data tables. GAB and DA wrote the manuscript. $\mathrm{GAB}, \mathrm{DA}, \mathrm{AB}$, and NP performed the analysis. MH contributed critical analytical tools for the analysis. CNMCM-W, DBK, KPF, MB, IK, and BY contributed citations and evaluated and edited the manuscript. GAB, DA, NP, and IK are guarantors of the data and analysis.

Funding: This study received no funding.

Competing interests: All authors have completed the ICMJE uniform disclosure form at www.icmje.org/coi_disclosure.pdf and declare: no support from any organization for the submitted work; no financial relationships with any organizations that might have an interest in the submitted work in the previous three years; no other relationships or activities that could appear to have influenced the submitted work.

Ethical approval: The deidentified data in this study were exempt from review by an institutional review board as confirmed by the Harvard Medical School institutional review board committee.

Data sharing: No additional data available.

Transparency: The manuscript's guarantors (GAB, DA, NP, and IK) affirm that the manuscript is an honest, accurate, and transparent account of the study being reported; that no important aspects of the study have been omitted; and that any discrepancies from the study as planned (and, if relevant, registered) have been explained.

This is an Open Access article distributed in accordance with the Creative Commons Attribution Non Commercial (CC BY-NC 4.0) license, which permits others to distribute, remix, adapt, build upon this work non-commercially, and license their derivative works on different terms, provided the original work is properly cited and the use is non-commercial. See: http://creativecommons.org/licenses/ by-nc/4.0/.

1 Rudd RA, Aleshire N, Zibbell JE, Gladden RM. Increases in Drug and Opioid Overdose Deaths--United States, 2000-2014. MMWR Morb Mortal Wkly Rep 2016;64:1378-82. doi:10.15585/mmwr. mm6450a3.

2 Elzey MJ, Barden SM, Edwards ES. Patient Characteristics and Outcomes in Unintentional, Non-fatal Prescription Opioid Overdoses: A Systematic Review. Pain Physician 2016;19:215-28. www.ncbi. nlm.nih.gov/pubmed/27228510.

3 Paulozzi LJ, Strickler GK, Kreiner PW, Koris CM. Centers for Disease Control and Prevention (CDC). Controlled Substance Prescribing Patterns--Prescription Behavior Surveillance System, Eight States, 2013. MMWR Surveill Summ 2015;64:1-14. doi:10.15585/mmwr. ss6409a1.

4 Centers for Disease Control and Prevention (CDC). Vital signs: overdoses of prescription opioid pain relievers---United States, 1999--2008. MMWR Morb Mortal Wkly Rep 2011;60:1487-92

5 Dunn KM, Saunders KW, Rutter CM. Overdose and prescribed opioids : Associations among chronic non-cancer pain patients. Ann Intern Med 2010;152:85-92. doi:10.7326/0003-4819-152 2-201001190-00006

6 Waljee JF, Zhong L, Hou H, Sears E, Brummett C, Chung KC. The Use of Opioid Analgesics following Common Upper Extremity Surgical Procedures: A National, Population-Based Study. Plast Reconstr Surg 2016;137:355e-64e. doi:10.1097/01. prs.0000475788.52446.7b

7 Bohnert ASB, Valenstein M, Bair MJ. Association between opioid prescribing patterns and opioid overdose-related deaths. JAMA 2011:305:1315-21. doi:10.1001/jama.2011.370.

8 Bicket MC, Long JJ, Pronovost PJ, Alexander GC, Wu CL. Prescription Opioid Analgesics Commonly Unused After Surgery: A Systematic Review. JAMA Surg 2017;152:1066-71. doi:10.1001/ jamasurg.2017.0831.

9 Waljee JF, Li L, Brummett CM, Englesbe MJ. latrogenic Opioid Dependence in the United States: Are Surgeons the Gatekeepers? Ann Surg 2017;265:728-30. doi:10.1097/ SLA.0000000000001904

10 Volkow ND, McLellan TA, Cotto JH, Karithanom M, Weiss SR. Characteristics of opioid prescriptions in 2009 JAMA 2011;305:1299-301. doi:10.1001/jama.2011.401

11 Menendez ME, Ring D, Bateman BT. Preoperative Opioid Misuse is Associated With Increased Morbidity and Mortality After Elective Orthopaedic Surgery. Clin Orthop Relat Res 2015;473:2402-12. doi:10.1007/s11999-015-4173-5. 
12 Hill MV, Stucke RS, McMahon ML, Beeman JL, Barth RJ Jr. An Educational Intervention Decreases Opioid Prescribing After General Surgical Operations. Ann Surg 2017;XX:1. doi:10.1097/ SLA.0000000000002198.

13 Wilkerson RG, Kim HK, Windsor TA, Mareiniss DP. The Opioid Epidemic in the United States. Emerg Med Clin North Am 2016:34:e1-23. doi:10.1016/j.emc.2015.11.002

14 Frieden TR, Houry D. Reducing the Risks of Relief--The CDC Opioid-Prescribing Guideline. N Engl J Med 2016;374:1501-4. doi:10.1056/NEJMp1515917.

15 Waxman BP. Medicine in small doses. ANZ J Surg 2015;85:210-1. doi:10.1111/ans.13026.

16 Clarke H, Soneji N, Ko DT, Yun L, Wijeysundera DN. Rates and risk factors for prolonged opioid use after major surgery: population based cohort study. BMJ 2014;348:g1251. doi:10.1136/bmj. g1251.

17 Sullivan MD. What are we treating with opioid and sedative-hypnotic combination therapy? Pharmacoepidemiol Drug Saf 2015;24:893-5. doi:10.1002/pds.3821.

18 Calcaterra SL, Yamashita TE, Min SJ. Opioid Prescribing at Hospital Discharge Contributes to Chronic Opioid Use. J Gen Intern Med 2016:31:38-43 doi:10.1007/s11606-015-3539-4.

19 Brummett CM, Waljee JF, Goesling J. New Persistent Opioid Use After Minor and Major Surgical Procedures in US Adults. JAMA Surg 2017;152:e170504. doi:10.1001/jamasurg.2017.0504.

20 Jena AB, Goldman D, Karaca-Mandic P. Hospital Prescribing of Opioids to Medicare Beneficiaries. JAMA Intern Med 2016;176 990-7. doi:10.1001/jamainternmed.2016.2737.

21 ACS. ACS National Surgical Quality Improvement Program. 2015. (accessed 1 Jan 2016)

22 Cerner Corporation. Multum Lexicon. 2016. www.multum.com/ Lexicon.htm (accessed 1 Jan 2016).

23 Medicaid $\mathrm{C}$ for $\mathrm{M}$ and. Opioid Morphine Equivalent Conversion Factors. 2014;1. www.cms.gov/Medicare/Prescription-DrugCoverage/PrescriptionDrugCovContra/Downloads/Opioid-MorphineEQ-Conversion-Factors-March-2015.pdf (accessed 7 Jan 2016).

24 Gomes T, Mamdani MM, Dhalla IA, Paterson JM, Juurlink DN. Opioid dose and drug-related mortality in patients with nonmalignant pain. Arch Intern Med 2011;171:686-91. doi:10.1001/ archinternmed.2011.117.

25 Mosher HJ, Richardson KK, Lund BC. The 1-Year Treatment Course of New Opioid Recipients in Veterans Health Administration. Pain Med 2016:17:1282-91. doi:10.1093/pm/pnw058.

26 Dasgupta N, Funk MJ, Proescholdbell S, Hirsch A, Ribisl KM, Marshall S. Cohort Study of the Impact of High-Dose Opioid Analgesics on Overdose Mortality. Pain Med 2016;17:85-98. doi:10.1111/pme.12907

27 Tibshirani R. Regression Shrinkage and Selection via the Lasso. / $R$ Stat Soc 1996;Series B:267-88.

28 Bateman BT, Choudhry NK. Limiting the Duration of Opioid Prescriptions: Balancing Excessive Prescribing and the Effective Treatment of Pain. JAMA Intern Med 2016;176:583-4. doi:10.1001/ jamainternmed.2016.0544.

29 Wunsch H, Wijeysundera DN, Passarella MA, Neuman MD. Opioids Prescribed After Low-Risk Surgical Procedures in the United States, 2004-2012. JAMA 2016:315:1654-7. doi:10.1001/ jama.2016.0130.

30 Edlund MJ, Martin BC, Russo JE, DeVries A, Braden JB, Sullivan MD The role of opioid prescription in incident opioid abuse and dependence among individuals with chronic noncancer pain: the role of opioid prescription. Clin J Pain 2014;30:557-64. doi:10.1097/ AJP.0000000000000021

31 Miller M, Barber CW, Leatherman S. Prescription opioid duration of action and the risk of unintentional overdose among patients receiving opioid therapy. JAMA Intern Med 2015;175:608-15 doi:10.1001/jamainternmed.2014.8071.
32 Bedard NA, Pugely AJ, Westermann RW. Opioid Use After Total Knee Arthroplasty: Trends and Risk Factors for Prolonged Use. I Arthroplasty. Published Online First, 2017.

33 Deyo RA, Hallvik SE, Hildebran C. Association Between Initial Opioid Prescribing Patterns and Subsequent Long-Term Use Among OpioidNaïve Patients: A Statewide Retrospective Cohort Study. / Gen Intern Med 2017;32:21-7. doi:10.1007/s11606-016-3810-3.

34 Sun EC, Darnall BD, Baker LC, Mackey S. Incidence of and Risk Factors for Chronic Opioid Use Among Opioid-Naive Patients in the Postoperative Period. JAMA Intern Med 2016;176:1286-93. doi:10.1001/jamainternmed.2016.3298.

35 Ladha KS, Patorno E, Huybrechts KF, Liu J, Rathmell JP, Bateman BT. Variations in the Use of Perioperative Multimodal Analgesic Therapy. Anesthesiology 2016;124:837-45. doi:10.1097/ ALN.0000000000001034.

36 Strassels SA, Chen C, Carr DB. Postoperative analgesia: economics, resource use, and patient satisfaction in an urban teaching hospital[table of contents.]. Anesth Analg 2002;94:130-7.

37 Morrison RS, Magaziner J, McLaughlin MA. The impact of post-operative pain on outcomes following hip fracture. Pain 2003;103:303-11. doi:10.1016/S0304-3959(02)00458-X.

38 Kehlet $\mathrm{H}$, Jensen TS, Woolf CJ. Persistent postsurgical pain: risk factors and prevention. Lancet 2006;367:1618-25. doi:10.1016/S01406736(06)68700-X.

39 Lenguerrand E, Wylde V, Gooberman-Hill R. Trajectories of pain and function after primary hip and knee arthroplasty: The adapt cohort study. PLoS One 2016;11:e0149306. doi:10.1371/journal. pone.0149306.

40 Wu CL, Raja SN. Treatment of acute postoperative pain. Lancet 2011:377:2215-25. doi:10.1016/S0140-6736(11)60245-6.

41 Volkow ND, McLellan AT. Opioid Abuse in Chronic Pain-Misconceptions and Mitigation Strategies. N Engl J Med 2016:374:1253-63. doi:10.1056/NEIMra1507771.

42 McCarty D, Janoff S, Coplan P, et al. Detection of opioid overdoses and poisonings in electronic medical records as compared to medical chart reviews. In: Presentation to FDA. 2014. www.fda.gov/ downloads/Drugs/NewsEvents/UCM398787.pdf

43 Hill MV, McMahon ML, Stucke RS. Wide Variation and Excessive Dosage of Opioid Prescriptions for Common General Surgical Procedures. Ann Surg 2016;XX:1-6. doi:10.1097/ SLA.0000000000001993.

44 Edlund MJ, Martin BC, Fan MY, Devries A, Braden JB, Sullivan MD. Risks for opioid abuse and dependence among recipients of chronic opioid therapy: results from the TROUP study. Drug Alcohol Depend 2010;112:90-8. doi:10.1016/j.drugalcdep.2010.05.017

45 Passik SD, Hays L, Eisner N, Kirsh KL. Psychiatric and pain characteristics of prescription drug abusers entering drug rehabilitation. J Pain Palliat Care Pharmacother 2006;20:5-13. www.ncbi.nlm.nih.gov/pubmed/16702131. doi:10.1080/ 1354v20n02 03

46 Ronan MV, Herzig SJ. Hospitalizations Related To Opioid Abuse/ Dependence And Associated Serious Infections Increased Sharply, 2002-12. Health Aff (Millwood) 2016;35:832-7. doi:10.1377/ hlthaff.2015.1424.

47 Ciesielski T, Iyengar R, Bothra A, Tomala D, Cislo G, Gage BF. A Tool to Assess Risk of De Novo Opioid Abuse or Dependence. Am J Med 2016;129:699-705.e4. doi:10.1016/j.amjmed. 2016.02.014

Supplemental information: ICD-9 codes of misuse (eTable1); all events Cox model (eTable2); CONSORT flow diagram (eFigure1); prescribing changes by sex, group, and surgery category (eFigure2); sensitivity analysis with one year events and no intervening surgery (eFigure3) 